\title{
Estudio exploratorio sobre la presencia de contaminantes en leche cruda proveniente de la cuenca lechera del Tarqui de la Sierra Sur Ecuatoriana
}

\author{
Maira Ortiz A. ${ }^{1}$, Cornelio Rosales J. ${ }^{1}$, Yolanda Aguilar V. ${ }^{1}$, Yury Murillo. ${ }^{1}$, Guillermo Serpa. ${ }^{1}$, \\ Tatiana Paguay N. ${ }^{2}$, Ángel G. Coronel A. ${ }^{2}$ \\ ${ }^{1}$ Facultad de Ciencias Agropecuarias, Universidad de Cuenca, Cuenca, Ecuador. \\ 2 Tesista, Universidad de Cuenca, Cuenca, Ecuador. \\ Autor para correspondencia: cornelio.rosales@ucuenca.edu.ec \\ Fecha de recepción: 24 de abril 2017 - Fecha de aceptación: 13 de junio 2017
}

\begin{abstract}
RESUMEN
La presente investigación determinó la existencia de contaminantes en la leche cruda de diez centros de acopio con capacidad de recepción entre 2,000 a 10,000 litros ubicados en siete cantones de la Provincia del Azuay. Se colectaron 90 muestras tomadas en tres visitas a cada centro durante los meses de julio y agosto de 2016. En cada muestra se analizó la presencia de antibióticos, peróxido de hidrógeno y neutralizantes mediante pruebas de diagnóstico rápido acreditadas en el laboratorio de inocuidad de alimentos de AGROCALIDAD, institución oficial de control de calidad. Para la detección de antibióticos se utilizó kit Trisensor ${ }^{\circledR}$ con sensibilidad de análisis validada a nivel mundial que permite detectar tres familias de antibióticos: betalactámicos, sulfonamidas y tetraciclinas según los límites máximos permitidos por el Codex Alimentarius. La detección de peróxido de hidrógeno se realizó mediante Test M-Quant ${ }^{\mathrm{TM}}$ que mide un rango entre $0.5 \mathrm{a} 25 \mathrm{mg}$ por litro de leche. La presencia de neutralizantes fue determinada mediante el método de alizarina, según la NORMA NTE INEN 1500:2011. Se analizaron 141,500 litros de leche cruda, en los que se detectó que en el 64.7\% de los casos la leche no era apta para el consumo humano, según lo establecido en la Norma NTE INEN 9:2012 y el Codex Alimentarius, por presencia de una o más de las sustancias estudiadas. Se encontró $13.3 \%$ de casos positivos a antibióticos betalactámicos y no hubo presencia de sulfonamidas y tetraciclinas; además, $16.4 \%$ de leche cruda se encontró adulterada con peróxido de hidrógeno y $60 \%$ con neutralizantes.
\end{abstract}

Palabras clave: Leche cruda, antibióticos, neutralizantes, conservantes, centros de acopio.

\begin{abstract}
The present survey aimed to define the presence of contaminants in the raw milk of ten collection centers, with a reception capacity between 2,000 and 10,000 liters, situated in seven cantons of the Province of Azuay. Ninety samples were collected during three visits to each of the collection centers during the months of July and August 2016. In each sample the presence of antibiotics, hydrogen peroxide and neutralizers was analyzed by means of rapid diagnostic tests accredited in the food safety laboratory of AGROCALIDAD, an official institution of quality control. For the detection of antibiotics, a Trisensor ${ }^{\circledR}$ kit with validated sensitivity, applied worldwide, was used to detect and evaluate the level of three antibiotic families: beta-lactams, sulfonamides and tetracyclines, versus the maximum permissible limits in the Codex Alimentarius. The detection of hydrogen peroxide was performed by Test M-Quant ${ }^{\mathrm{TM}}$ measuring a range between 0.5 to $25 \mathrm{mg}$ per liter of milk. The presence of neutralizers was determined by the alizarin method according to NTE NTE INEN 10:2012. 141,500 liters of raw milk were analyzed of which $64.7 \%$ were not fit for human consumption for the presence of one or more of the studied substances according to the NTE INEN Standard 9:2012 and the Codex Alimentarius. $13.3 \%$ of the samples were positive for beta-lactam antibiotics, but sulfonamides and tetracyclines was not detected. Besides $16.4 \%$ of the raw milk was adulterated with hydrogen peroxide and $60 \%$ with neutralizers.
\end{abstract}


Keywords: Raw milk, antibiotics, neutralizing, preservative, collection center.

\section{INTRODUCCIÓN}

La determinación de contaminantes presentes en la leche cruda bovina es un tema de importancia para la salud pública a nivel mundial ya que la leche y sus derivados constituyen productos de consumo masivo, destinándose aproximadamente el $75 \%$ de la producción para el consumo humano. Ecuador registra una producción diaria de 6,262,408 litros de leche, y de esta, la Provincia del Azuay aporta con una producción diaria de 583,669 litros, lo cual corresponde al 9.32\% del total nacional (Instituto Nacional de Estadísticas y Censos, 2013). En el Azuay 344,353 litros diarios de leche, que corresponden al 59\%, son transportados desde la finca a centros de acopio, previo a llegar a la industria lechera.

El artículo 13 de la Constitución de la República del Ecuador establece que las personas y colectividades tienen derecho al acceso seguro y permanente a alimentos sanos, suficientes y nutritivos, por lo que es importante garantizar la comercialización de leche sana e inocua. El no cumplimiento con lo establecido acarrea riesgos en la salud de los consumidores ya que el consumo o uso de leche con residuos de antibióticos, provenientes de fármacos de uso veterinario, pueden provocar alergias, toxicidad directa y resistencia bacteriana (Chamorro et al., 2010). Por otra parte, la presencia de antibióticos también dificulta los procesos industriales de elaboración de quesos, yogures y otros derivados cuyos procesos implican la fermentación con cultivos bacterianos (Vásquez \& Olivera, 2012). Las soluciones diluidas de peróxido de hidrógeno pueden inducir vómitos, leve irritación gastrointestinal, distensión gástrica (Agency for Toxic Substances and Disease Registry, 2002) y mutaciones (New Jersey Department of Health, 2008), además de causar problemas en la elaboración de derivados lácteos (Villegas de Gante \& Santos Moreno, 2013). Calderón et al. (2013) consideran que altas concentraciones de carbonatos o bicarbonatos en el cuerpo humano pueden interrumpir las señales hormonales que regulan el desarrollo y la reproducción.

A pesar de que la Norma NTE INEN 9:2012 (Instituto Ecuatoriano de Normalización, 2012a) menciona con claridad las sustancias prohibidas con propiedad neutralizante (orina, carbonatos, hidróxido de sodio y jabones) y conservante (formaldehído, peróxido de hidrógeno, cloro, hipocloritos, cloraminas, lactoperoxidasa adicionada y dióxido de cloro), es conocido que en la cadena de comercialización de la leche operan intermediarios que acopian leche cruda para venderla de manera directa al consumidor final o entregar a otros centros de acopio. En estos casos es práctica común adicionar sustancias que prolongan el tiempo de conservación de la leche ya sea por neutralización de la acidez de la leche o impidiendo la acidificación normal de la misma, contaminando de esa manera el producto y de esta manera generando problema de salud pública.

Por lo dicho, la investigación tuvo el objetivo de determinar la presencia de residuos de antibióticos (betalactámicos, tetraciclinas y sulfonamidas), de peróxido de hidrógeno y de neutralizantes de manera general y mediante pruebas de diagnóstico rápido, aplicadas en la leche cruda almacenada en los centros de acopio de mayor capacidad de recepción, ubicados en la más importante cuenca lechera de la Sierra Sur de la provincia del Azuay.

\section{MATERIALES Y MÉTODOS}

\subsection{Materiales}

La investigación se desarrolló en siete cantones de la provincia del Azuay ubicada al sur del Ecuador. La superficie abarcada por el estudio tiene $6,155 \mathrm{~km}^{2}$, una población de 602,429 habitantes y su cabecera provincial es la ciudad de Cuenca (Gobierno Provincial del Azuay, 2011). Para esta investigación se consideró el $100 \%$ de centros de acopio de leche cruda con capacidad individual de recepción de entre 2,000 y 10,000 litros, los cuales se hallan registrados en el organismo oficial de control, mediante resolución DAJ-2013461, y que cuentan con una capacidad total de acopio de 57,300 litros diarios. El 
total de leche analizada de la cual fueron tomadas muestras en el periodo de investigación (meses de julio y agosto de 2016) fue de 141,300 litros correspondientes a 10 centros de acopio.

\subsection{Métodos}

Se tomaron tres muestras del pool de leche cruda acopiada en los centros de acopio antes mencionados, realizándose tres visitas con quince días de intervalo entre ellas. Para el muestreo se usó un cucharón de acero inoxidable, extrayendo un volumen de $40 \mathrm{ml}$ directamente del tanque de enfriamiento. Posteriormente estas muestras se colocaron en envases adecuados e identificados para el propósito, se cerraron herméticamente y se transportaron al laboratorio en un termo de refrigeración a una temperatura de 2 a $4^{\circ} \mathrm{C}$ para su respectivo análisis. Para la detección de antibióticos se usó el método Trisensor ${ }^{\circledR}$ (test para la detección de residuos de betalactámicos, tetraciclinas y sulfamidas simultáneamente en una misma muestra de leche), para peróxido de hidrógeno el método Merckoquant ${ }^{\mathrm{TM}}$ (prueba que mide un rango entre 0.5 a $25 \mathrm{mg}$ por litro de leche) y para la determinación de neutralizantes se utilizó la prueba de alizarina (Instituto Ecuatoriano de Normalización, 2012b). Estas son las pruebas aceptadas oficialmente ya sea por el organismo de control de calidad o por la norma técnica nacional.

\subsection{Diseño experimental}

La investigación fue de carácter descriptivo, observacional y transversal lo que permitió describir la situación de la calidad de la leche cruda en un determinado momento bajo la consideración de la existencia de contaminantes en la misma. Para la presentación de resultados se utilizaron cuadros y gráficos de frecuencias.

\section{RESULTADOS}

A partir de la información recolectada se pudo determinar que del total de muestras, obtenidas en los diferentes momentos, el $66.7 \%$ presentaron positividad a cualquiera de las variables analizadas, es decir residuos de antibióticos, peróxido de hidrógeno y/o neutralizantes. Esto significa que el $64.7 \%$ de la leche acopiada estuvo contaminada (Fig. 1, sección izquierda). Se observó que a excepción del cantón
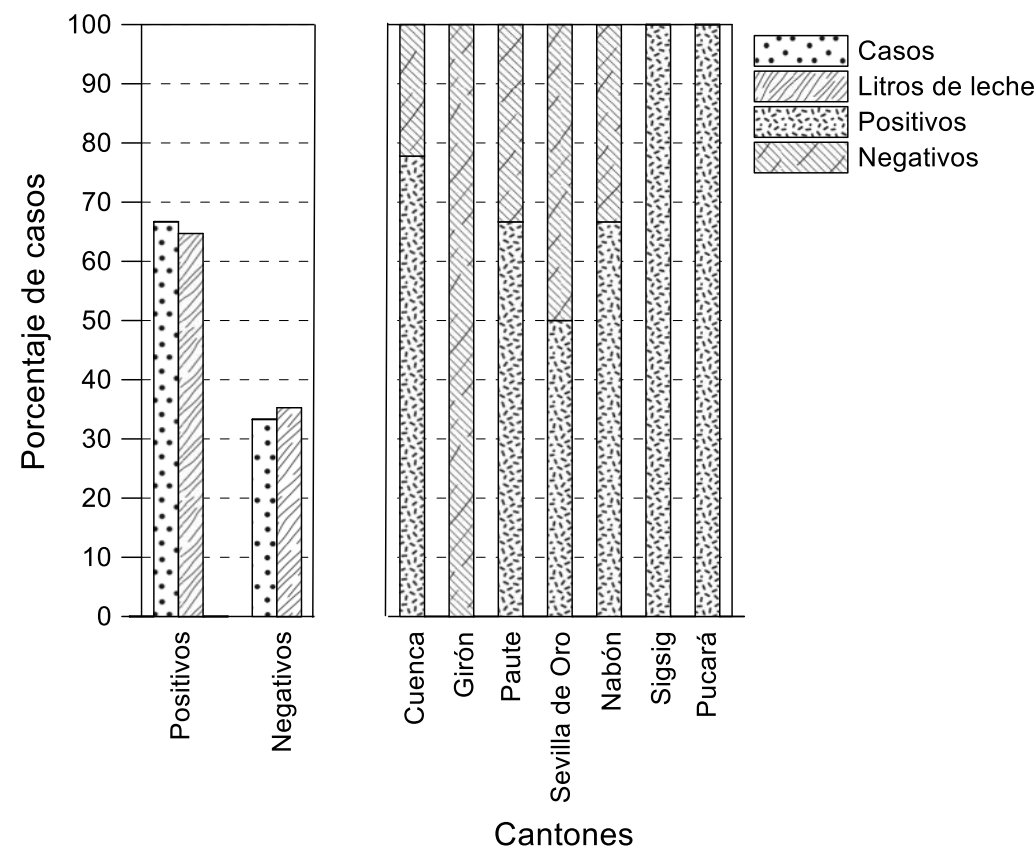

Figura 1. Porcentaje de casos positivos a contaminantes en leche cruda total (sección izquierda) y por cantón (sección derecha). 
Girón, que no presentó casos positivos, el resto de unidades territoriales presentaron porcentajes superiores al 50\% de casos positivos encontrándose en dos de ellos 100\% de positividad (Fig. 1, sección derecha).

Desagregados los casos positivos por contaminante, se encontró que el $40 \%$ de centros de acopio y el $13 \%$ de muestras dieron resultados positivos a antibióticos, el $40 \%$ y el $17 \%$ a peróxido de hidrógeno y el $90 \%$ y el $60 \%$ a sustancias neutralizantes (Fig. 2).

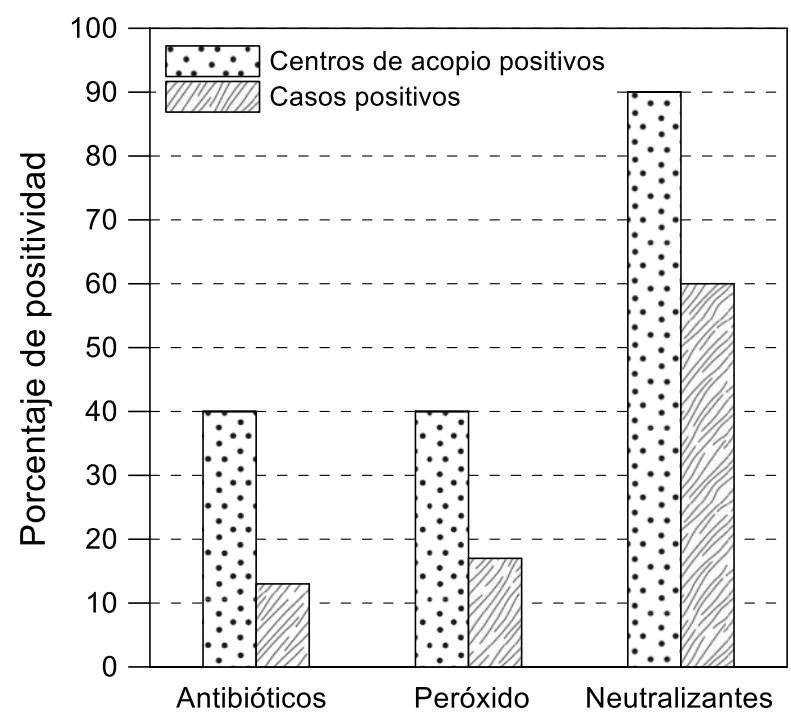

Figura 2. Porcentaje total de centros de acopio y casos reacciones positivas de acuerdo al tipo de contaminante en leche cruda.

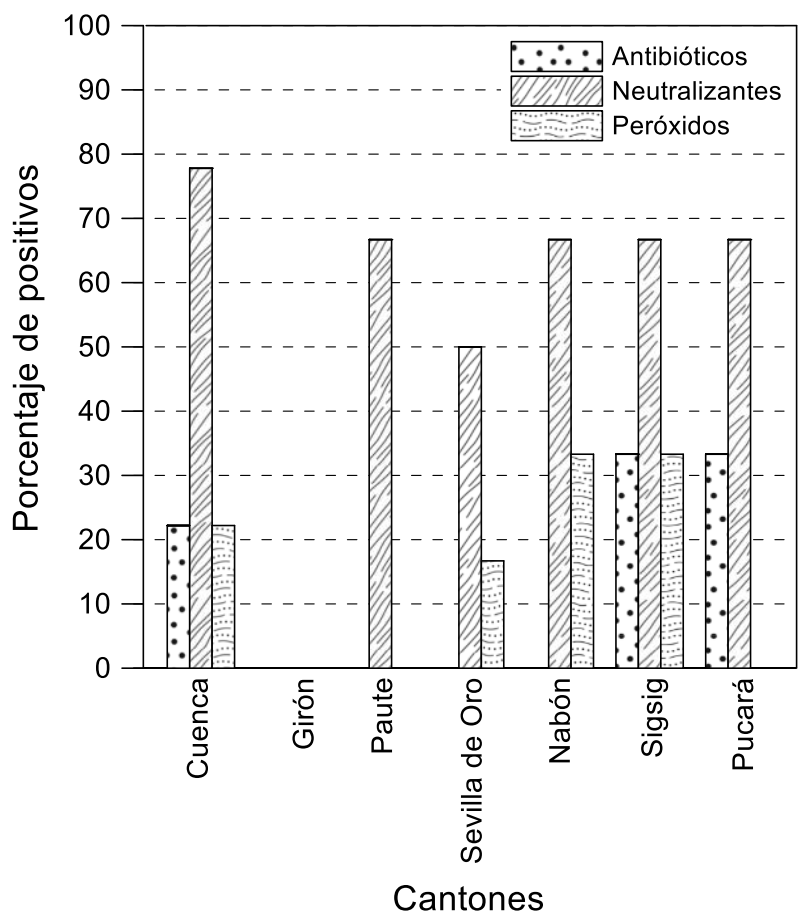

Figura 3. Porcentaje de reacciones positivas de acuerdo de área geográfica y tipo de contaminante en leche cruda.

De acuerdo al área territorial de ubicación de los centros de acopio, se encontró que los cantones de Sígsig, Pucará y Cuenca registraron presencia de antibióticos en la leche acopiada, observándose, respectivamente, $33 \%, 33 \%$ y $22.2 \%$ de casos positivos para antibióticos betalactámicos. No se 
observaron reacciones positivas a otro tipo de antibióticos. En cuanto a la presencia de peróxido de hidrógeno, se observó que en Sígsig, Nabón, Cuenca y Sevilla de Oro se encontraron el 33.3\%, 33.3\%, $22.2 \%$ y $16.7 \%$ de casos positivos respectivamente. Por último, con respecto a la presencia de neutralizantes, el cantón con mayor incidencia fue Cuenca con $77.8 \%$ de muestras positivas, seguido por Paute, Nabón, Sigsig y Pucará con un porcentaje de $66.7 \%$ cada uno y Sevilla de Oro con 50\% de muestras positivas (Fig. 3).

\section{DISCUSIÓN}

La leche cruda analizada presenta positividad a antibióticos del tipo betalactámicos en un $13 \%$ del total analizado, proveniente del $40 \%$ de centros de acopio, lo que implica la no aceptabilidad de dicho producto para el consumo humano de acuerdo a la norma del Instituto Ecuatoriano de Normalización NTE INEN 9:2012, derivada de los límites máximos permitidos (MRL) establecidos por el Codex Alimentarius CAC/MRL 2 (Organización de las Naciones Unidas para la Alimentación y la Agricultura \& Organización Mundial de la Salud, 2015). En un estudio similar en los mercados de Perú Guerrero et al. (2009), utilizando el método presuntivo para determinación de antibióticos betalactámicos y tetraciclinas, encontró un $40 \%$ de muestras positivas, siendo este un porcentaje muy superior al encontrado en la presente investigación. Torres \& Pillco (2013) en su estudio en Cuenca (Ecuador), encontraron el 3.23\% de leche con residuos de antibióticos sin especificar su tipo; por su parte Chamorro et al. (2010), en el sector urbano del Municipio de Ipiales, encontraron el 5\% de muestras positivas al mismo grupo de antibióticos, en ambos casos valores inferiores a los encontrados en este estudio. La realidad determinada en nuestro caso muy probablemente se debe al uso indiscriminado y sin control de antibióticos del grupo betalactámicos para el tratamiento de infecciones generales y de la glándula mamaria en particular, como lo sugieren Vásquez \& Olivera (2012), Guerrero et al. (2009) y Ramírez et al. (2012), quienes coinciden en varias de las fuentes de contaminación con residuos de antibióticos como son el tiempo de retiro inadecuado, olvido de retiro de la leche, uso de fármacos para secado pocos días antes del parto, retiro de la leche solo del cuarto tratado, sobredosificación y uso indiscriminado de antibióticos sin control y prescripción médica (Máttar et al., 2009). Cabe agregar que este tipo de antibióticos son de los más utilizados en la práctica veterinaria general, por lo cual no es extraño encontrar niveles de contaminación tan altos. De acuerdo con esto, estos contaminantes no son agregados directamente a la leche, sino que por lo general son metabolitos residuales presentes después de ser utilizados para el tratamiento de procesos patológicos del animal, esto lo demostraron Ramírez et al. (2012) al encontrar residuos de amoxicilina hasta 84 horas y la asociación cloxacilina-amoxicilina 60 horas post-tratamiento.

Por el contrario, el peróxido de hidrógeno asegura la conservación ilícita de la leche. Su uso está prohibido ya que inhibe la microbiota láctica que produce la acidificación natural. El 17\% de muestras analizadas pertenecientes al $40 \%$ de centros de acopio resultaron positivas, por lo que dicha leche no es aceptable para consumo humano, de acuerdo a lo establecido en la norma NTE INEN 9:2012 y el CODEX ALIMENTARIUS. Lo encontrado difiere con los estudios realizados por otros autores en Venezuela (Rondón et al., 2003) y en Guayaquil (Carpio, 2001) quienes no encontraron presencia de residuos de peróxido de hidrógeno. Nuestros hallazgos sugieren que existe uso ilegal de la mencionada sustancia, debido a la existencia importante de nichos de mercadeo tradicionales e informales que, por sus limitados o inexistentes controles, receptan leche sin pruebas de control de calidad para la producción de quesos y/o expendio de leche cruda para consumo sin pasteurizar.

En lo que respecta a neutralizantes, su uso tiene como finalidad neutralizar el ácido láctico producido por la fermentación de la lactosa a través de microorganismos específicos. La investigación indica un 60\% de muestras positivas provenientes del $90 \%$ de centros de acopio estudiados, lo que fue muy próximo a lo encontrado por Rondón et al. (2003) que observó el 80\% de muestras con presencia de neutralizantes. Por el contrario, Torres \& Pillco (2013) y Calderón et al. (2013) solo pudieron encontrar, respectivamente, el $8.6 \%$ y $21.21 \%$ de muestras positivas. La alta prevalencia encontrada en el uso de neutralizantes posiblemente se debe a una mala rutina de lavado de los utensilios de ordeño por lo que quedan residuos de detergentes o jabones comúnmente usados para uso doméstico. 


\section{CONCLUSIÓN}

El 64.7\% de la leche cruda analizada en esta investigación no fue apta para el consumo humano, esto representa un alto riesgo para la salud pública ya que incumple con lo establecido en la norma NTE INEN 9:2012 y el CODEX ALIMENTARIUS, al mostrar presencia de residuos de antibióticos betalactámicos, neutralizantes y/o peróxido de hidrógeno. A diferencia de la leche proveniente de Girón, cuyas muestras analizadas no evidenciaron presencia de contaminantes, las unidades territoriales de Sígsig y Pucará fueron las más afectadas al presentar un $100 \%$ de muestras contaminadas con alguna o todas las sustancias investigadas. En cuanto a los centros de acopio en estudio, solamente uno cumplió con los requisitos establecidos en las mencionadas normas para las tres sustancias analizadas.

Los resultados encontrados nos permiten deducir la existencia de: un uso indiscriminado y sin control de antibióticos betalactámicos, el uso de peróxido de hidrógeno de manera ilegal para ubicar leche en mercados informales y, la contaminación de la leche con detergentes domésticos usados para la higiene de utensilios de ordeño y enjuagados deficientemente. Frente a estos datos, el mejoramiento de los sistemas de control oficial y la capacitación, a todos los que intervienen en la cadena de comercialización de la leche, es apremiante. También, es necesario realizar un diagnóstico que permita visualizar la realidad actual sobre las buenas prácticas en la producción agropecuaria y confirmar los resultados positivos con el uso de cromatografía líquida de alta eficacia (HPLC).

\section{BIBLIOGRAFÍA}

Agency for Toxic Substances and Disease Registry (ATSDR) (2002). Peróxido de hidrógeno. Disponible en https://www.atsdr.cdc.gov/toxfaqs/tfacts174.pdf, 2 pp

Calderón A, Rodríguez V, Martínez N (2013). Determinación de adulterantes en leches crudas acopiadas en procesadoras de quesos en Montería (Córdova). ORINOQUIA 17(2):202206

Carpio L (2001). Factores asociados a la adulteración comercial de leches y yogures en Guayaquil. Tesis de doctorado, Universidad de Guayaquil, Guayaquil, Ecuador, 124 pp. Disponible en http://repositorio.ug.edu.ec/bitstream/redug/706/ 1/LECHE\%20y\%20yogurt.pdf

Chamorro J, López E, Astaiza J, Benavides C, Hidalgo A (2010). Determinación de la calidad composicional y de residuos antibióticos. Revista Centro De Estudios en Salud 1(12):89-101

Gobierno Provincial del Azuay (2011). Plan de desarrollo y ordenamiento territorial de la Provincia del Azuay actualizado 2015-2030. Disponible en http://app.sni.gob.ec/snilink/sni/PORTAL_SNI/data_sigad_plus/sigadplusdocumentofinal/ 0160000190001_PDyOT_AZUAY\%20\%202015_17-08-2015_10-02-34.pdf, 308 pp

Guerrero D, Gamarra G, Benavides E, Roque M, Salazar M (2009). Detección de residuos de antibióticos betalactámicos y tetraciclinas en leche cruda comercializada en el Callao. Ciencia e Investigación 12(2):79-82

Instituto Ecuatoriano de Normalización (2012a). Leche cruda. Requisitos. Norma Técnica Ecuatoriana NTE INEN 9:2010 ( $1^{\text {a }}$ ed., $5^{\text {a }}$ revisión). Disponible en https://archive.org/stream/ (c.nte.0009.2008/ec.nte.0009.2008_djvu.txt, 8 pp

Instituto Ecuatoriano de Normalización (2012b). Leche pasteurizada. Requisitos. Norma Técnica Ecuatoriana NTE INEN 10:2012 (1 ${ }^{\mathrm{a}}$ ed., $5^{\mathrm{a}}$ revisión). Disponible en http://normaspdf.inen.gob.ec/pdf/nte/10-5.pdf, 10 pp

Instituto Nacional de Estadísticas y Censos (2013). Encuesta de superficie y producción agropecuaria continua ESPAC-2013. Disponible en 
http://www.ecuadorencifras.gob.ec/encuesta-de-superficie-y-produccion-agropecuariacontinua-espac-2013/

Máttar S, Calderón A, Sotelo D, Sierra M, Tordecilla G (2009). Detección de Antibióticos en Leches: Un Problema de Salud Pública. Revista de Salud Pública Universidad Nacional de Colombia 11(4):579-590

New Jersey Department of Health (NJ Health) (2008). Peróxido de Hidrógeno. Disponible en http://www.nj.gov/health/

Organización de las Naciones Unidas para la Alimentación y la Agricultura \& Organización Mundial de la Salud (2015). Residuos de medicamentos veterinarios en los alimentos. Codex alimentarius, Normas internacionales de los alimentos, CAC/MRL 2. Retrieved from http://www.fao.org/fao-who-codexalimentarius/standards/vetdrugs/es/

Ramírez G, Vélez G, Rondón I (2012). Determinación de residuos de antibióticos y tiempo de retiro en leche proveniente del Municipio de Cartago (Valle del Cauca). Revista Colombiana de Ciencia Animal 5(1):25-31

Rondón L, Lara E, González I (2003). Agentes adulterantes y conservadores en la leche fluida. Revista de la Facultad De Farmacia 45(2):45-50

Torres A, Pillco V (2013). Calidad fisico-química de la leche cruda que ingresa a la ciudad de Cuenca, para su comercialización. Tesis de pregrado, Facultad de Ciencias Químicas, Universidad de Cuenca, Cuenca, Ecuador, 97 pp

Vásquez J, Olivera M (2012). Residuos de $\beta$-lactamicos en leche cruda y factores asociados a su presentación. Revista UDCA Actualidad \& Divulgación Científica 15(1):157-165

Villegas de Gante A, Santos Moreno A (2013). Calidad de leche cruda. (1 ${ }^{\mathrm{a}}$ ed.). México DF, México: Editorial Trillas S.A. de C.V, 100 pp 